\title{
Research on Random Vibration Control Algorithm Based-on EV Model
}

\author{
Yu Haoyang ${ }^{1, a}$, Guan Guangfeng ${ }^{2, b}$, Feng Chenlu ${ }^{1, a}$
}

1. Depart of Electronic Engineering Heilongjiang Institute of Technology Harbin, 150050, China

2. Mechanical Engineering Department Dalian Maitime University116026, China

achina-yhy-111@163.com, ${ }^{b}$ guanguangfeng@yahoo.com.cn

Keywords: random vibration; EV model; FRF estimation; PSD (power spectral density)

\begin{abstract}
In order to improve the accuracy of random vibration power spectrum playback experiment, The traditional FRF H1 estimation has improved. The methods of FRF estimation have been proposed based on EV model for the H1 under-estimation problems. The FRF H1 estimator is updated based on the power spectral density (PSD) of the noises and inputs of the system, and describes the principle and process of random vibration control algorithm based on EV model, and finally the acceleration power spectrum playback experiment verify the validity of the algorithm.
\end{abstract}

\section{Introduction}

Vibration experiments aimed at evaluating the ability of the product to maintain the original performance under vibration stimuli circumstance, research and test the reliability of the product structure and manipulation in the vibration condition. Hydraulic vibration table is used in large-scale engineering structure or the model and real vibration simulation experiment ${ }^{[1]}$. In order to improve the control precision, reproduce the reference signal more accurately and form a true sense of the vibration of closed-loop control, the driven signal is compensated through the vibration control to reduce the difference of the response signal and reference signal ${ }^{[2]}$.

FRF estimation is the basis of random vibration Control, whose estimation results are directly related to precision of vibration control ${ }^{[3]}$.

This article sketches the FRF H1 estimation and analyses its Under-Estimation. Then propose the FRF model identification system and present random vibration control algorithm the frequency response function estimates based on the EV model. Finally, the acceleration power spectrum playback experiment verified the effectiveness of the algorithm.

\section{Based on The EV Model of Frequency Response Function Estimation}

RF H1 estimation assumes there is no noise in signal input terminal. In the vibration control system, input signal is referred to as the driving signal, the output signal is called the control signal. Let $d(t)$ is the system drive signal, $\eta(t)$ is an output noise signal, $c(t)$ is the control signal. FRF H1 estimation function is as follows ${ }^{[4]}$ :

$$
H_{1}(f)=G_{c d}(f)\left[G_{d d}(f)\right]^{-1}
$$

Where, $G_{d d}(f)$ is the spectral densities estimate of the driving signal, $G_{c d}(f)$ is the cross-spectral density estimate of the driving signal and control signal.

Because of the noise in the input signal, the $G_{d d}(f)$ estimation results will contain the power spectrum density of the noise signal, so the $\mathrm{H} 1$ estimation method is the under-estimation of the real frequency response function of system.

EV model assumes that noise exists in the system both the signal input and output terminal, its system model is shown in Fig.1:

In the figure, $u(t)$ is the actual drive signal of the system, $v(t)$ is the actual control signal, $\varepsilon(t)$ for the noise signal of the input . $\varepsilon(t)$ and $\eta(t)$ are unrelated, and they are all mutually uncorrelated with drive signals and control signals. From the H1 estimation method we can get the real system frequency response function, which 


$$
H_{0}(f)=G_{u v}(f)\left[G_{u u}(f)\right]^{-1}
$$

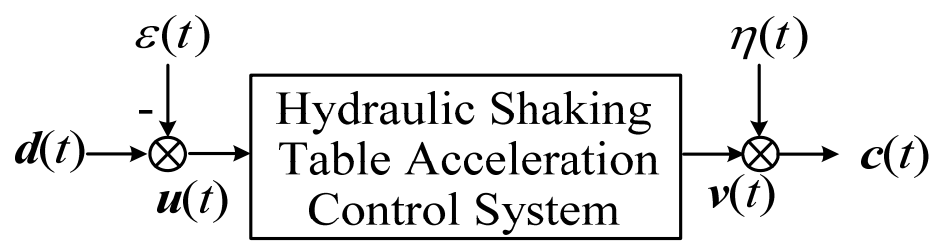

Figure 1 shows

Fig. 1 The EV model of the system

$\left\{\begin{array}{l}d(t)=u(t)-\varepsilon(t) \\ c(t)=v(t)+\eta(t)\end{array}\right.$

We can learn from formula (3), the spectral densities estimate is,

$G_{d d}(f)=G_{u u}(f)-2 G_{u \varepsilon}(f)+G_{\varepsilon \varepsilon}(f)$

As $\varepsilon(\mathrm{t})$ and $\mathrm{u}(\mathrm{t})$ are unrelated, cross-spectral density value is approximately zero. So Eq.4 can be simplified to

$$
G_{d d}(f)=G_{u u}(f)+G_{\varepsilon \varepsilon}(f)
$$

Similarly, we have

$$
G_{c d}(f)=G_{u v}(f)
$$

(6)

Take formula (5) and (6) into (1), and use (2) to finishing, here is (7)

$H_{0}(f)=H_{1}(f)\left(1+\left[G_{u u}(f)\right]^{-1} G_{\varepsilon \varepsilon}(f)\right)$

$G_{u u}(f)$ and $G_{\varepsilon \varepsilon}(f)$ is known, then use the formula(7) to get the real system frequency response function.

\section{Random Vibration Control Algorithm}

Random vibration control algorithm schematic based on EV model the frequency response function estimates are shown in Figure.3:

Figure 3 shows that the random vibration control algorithm is mainly composed of Frequency response function estimates, impedance calculation,driving spectrum correction and drive signal generation.

Frequency Response Function Estimation.Let $d(t)=0$, then the measured actual driving signal $u(t)$ of system is the input noise signal $\varepsilon(\mathrm{t})$, using Welch method to estimate the spectral density $G_{\varepsilon \varepsilon}(f)$ of the noise signal.

Where band-limited white noise is driven signal $d(t)$,measure the real driven signal $\mathrm{u}(\mathrm{t})$ and control signal $\mathrm{c}(\mathrm{t})$. Use formula (7) to calculate the results of frequency response function estimation based on the EV model .

Impedance Calculation.System impedance is the inverse of the frequency response function

$$
Z(f)=\left[H_{0}(f)\right]^{-1}
$$

Driving Spectrum Correction.Make $\mathrm{R}(\mathrm{f})$ as reference spectrum signal, the definition of the spectral density of control signal after the $\mathrm{j}$-th iteration is the $\mathrm{Gcc}(\mathrm{f})$, then the error spectrum is,

$$
E(f)_{j}=R(f)-G_{c c}(f)_{j}
$$

Iterative formula is corrected by drive spectrum,

$$
G_{d d}(f)_{j+1}=G_{d d}(f)_{j}+O Z_{0}(f) E(f)_{j}\left[Z_{0}(f)\right]^{H}
$$

Where, $\operatorname{Gcc}(f) j+1$ is spectral density of drive signal after the $(j+1)$-th iterative computation, $\alpha$ is the iteration step. When $0<\alpha<1$, formula (10) is convergent ${ }^{[5]}$..

Drive Signal Generation.The driving spectrum after modifying is signal infrequency domain, it needs to be transformed signal in time domain can it drive system, the Figure 4 gives the schematic diagram that is the process of the driving spectrum signal transform to the driving time domain signal. 




Fig. 3 Random vibration control algorithm based on EV model the FRF estimates.

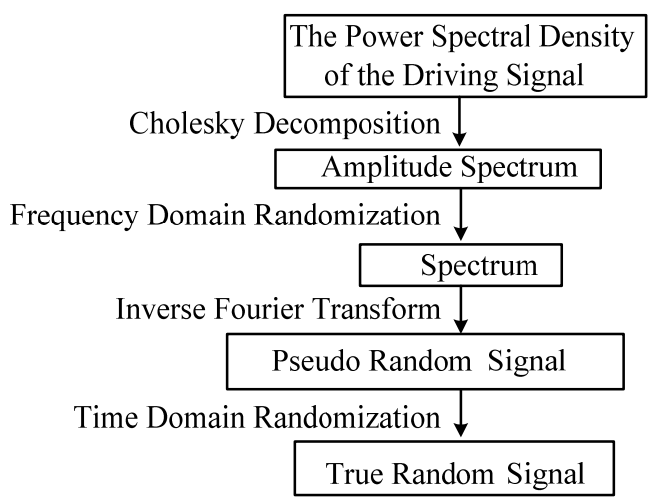

Fig.4 The time domain random signal generation process

1)Here's amplitude spectrum : Convert the driving spectrum signal into amplitude spectrum signal by Cholesky transform, namely

$\boldsymbol{G}_{d d}(f)=\boldsymbol{D}^{\prime}(f) \boldsymbol{D}^{\prime}(f)^{H}$

In the formula, $D^{\prime}(f)$ is the amplitude spectrum, only frequency information, no phase information.

2)Here's random phase: Use the frequency domain randomization to add a Gaussian distributions random phase, here comes

$$
\mathbf{D}(f)=\mathbf{D}^{\prime}(f) e^{j \theta_{i}} \quad i=1, \ldots, n
$$

In the formula, $D(f)$ is the frequency spectrum, $\theta_{i}$ is Gaussian-like distribution random phase, $n$ is the frame length of the inverse fourier transform .

3)Inverse Fourier transform: transform spectrum signal which is getting from the frequency domain randomization to a frame random signal converted from inverse Fourier transform, namely

$$
d(t)=\operatorname{IFFT}[D(f)]
$$

The power spectrum density of a frame random signal converted from inverse Fourier transform is the driving spectrum density.

4)The product of the true -random signal: Only considering the corresponding relation of the time domain signal and the density of the driving signal that we can use the cycle repetition of the a frame by inverse fourier transforming as the driving time domain signal, but it has two disadvantages: firstly, it is the problem of the frequency leakage: due to the multiple repeated a frame is the pseudo-random signal in essence, its spectrum is the discrete spectrum, and the power concentrates on the frequency point of the primary spectrum , so the frequency leakage is more serious . Secondly, it is the dovetail-problem of the all frame signals dovetail-problem: a frame time domain signal by inverse fourier transform generating, its starting point and ending point are all zero value, in this way it will cause connected multi frame signals product step signals, they will lead a huge impact..even each frame signals' starting point and ending point are all zero value, can't we guarantee the continuous derivability of the signals after the connecting, so it also can cause impact.

In order to generate the true random signal, make the inverse Fourier transform random a frame signal after random delay, reverse, add window and stack processed by turns to become true random signal, this process is called the time domain random process ${ }^{[6] .}$.

Make a data frame signal which is converted from inverse Fourier transform connected end to end, taken from one point as the starting point randomly, take n-samples sequence as a Data Frame signal in order or reverse。Repeat the above process, you can get $2 \mathrm{n}$ sequences。Then make them together in accordance with the ratio of 3:1which were through the dispose adding window, the signal is the real random signal.

Regard the generated signal by the time domain randomization as the input signal to drive 
hydraulic vibration table acceleration control system, the response signal of the measurement system and estimate response spectrum. Testing the precision of the power spectrum reappearance, if it meets the test requirements, we can stop iterating. Otherwise, we need to continue iterate.

\section{Experiment Research}

On the hydraulic cylinder of the hydraulic vibration table which is described, we can see the reappearance experiment of random vibration acceleration power spectrum density.

The figure 5 shows us H1 estimation method and the hydraulic cylinders' acceleration amplitude-frequency characteristic contrasting pattern whose obtained based on EV model frequency response function estimation method.. From the figure, we can see the result of the frequency response function'samplitude frequency characteristic whose obtained is based on the EV model is bigger than the result that is based on the $\mathrm{H} 1$ estimation method. It keeps consistent with aforesaid analysis results.

We use the reference spectrum's acceleration spectrum density which is between 0.5 and $2 \mathrm{HZ}$ as increased spectrum, $2 \sim 100 \mathrm{HZ}$ is straight spectrum, and the power spectrum density of straight spectrum is $3 \times 10-4 \mathrm{~g} 2 / \mathrm{Hz}$ 。

The figure 6 shows use the power spectrum contrasting pattern between the exciting spectrum whose obtained based on the EV model's the first iterative in the random vibration's control algorithm and the corresponding power spectrum of the driving time domain signal.

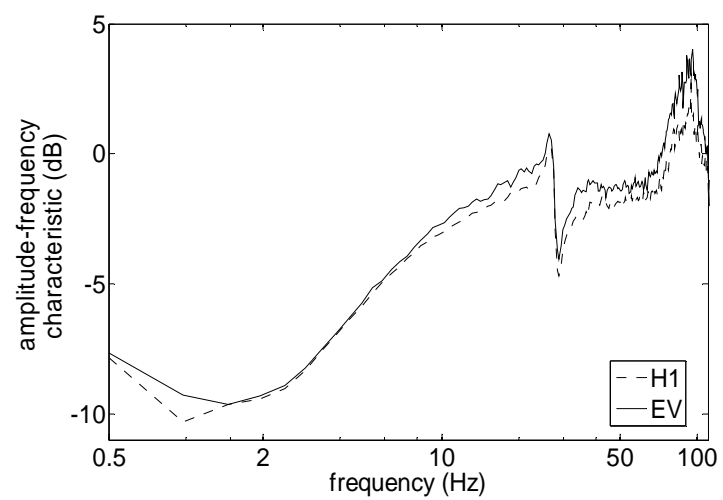

Fig. 5 The amplitude-frequency characteristic Of the frequency response function

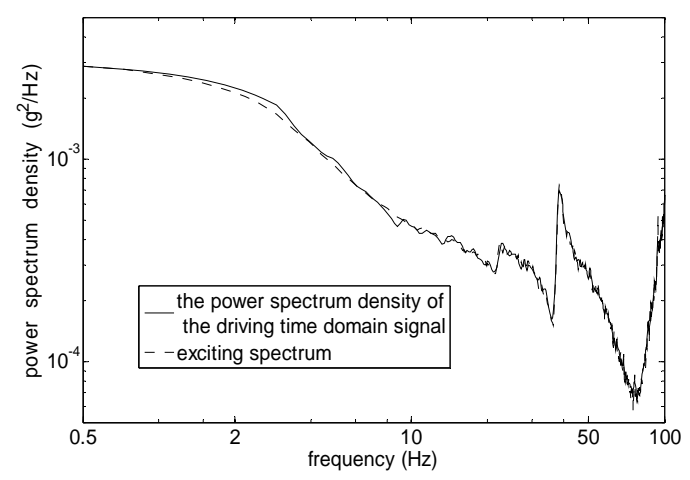

Fig.6 The power spectrum density of the driving time domain signal

From the figure 6 , we know ,the power spectrum of the driving time domain signal is matched with the exciting spectrum. It can prove the driving time domain signal's production method described in the 2-4 segment is effective.

The figure 7 shows the acceleration spectrum density test 's curve, it is the curve after twice iterating by the frequency response function $\mathrm{H} 1$ estimation method, the figure 8 shows the acceleration spectrum density test 's curve which is based on the frequency response function of the model estimation frequency response function after twice iterating. The $\pm 3 \mathrm{~dB}$ tolerance limit of the reference spectrum in the figure 7 and 8 is hydraulic vibration table hydraulic vibroplatform random vibration's precision requirement, and it is regulated by the national standard ${ }^{[7]}$. 


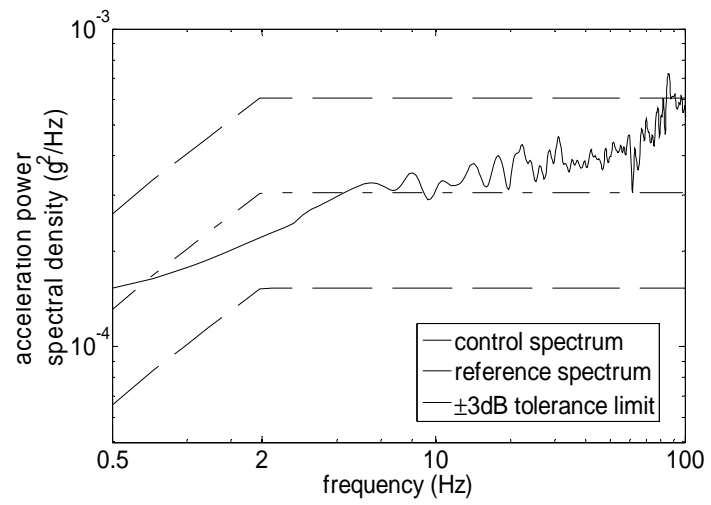

Fig.7 Reappearance test curve of the acceleration power spectral by using $\mathrm{H} 1$ estimation method

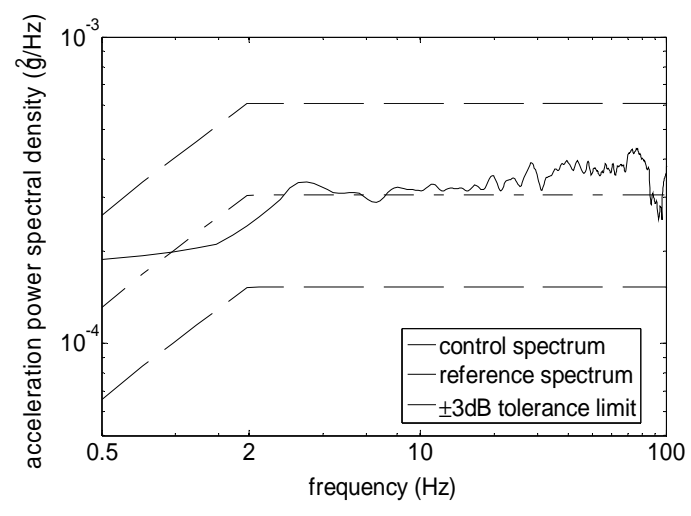

Fig.8 Reappearance test curve of the acceleration power spectral by using EV model

Compared figure 7 with figure 8 , we can know, with the same iteration number,when we use H1 estimates frequency response function, control spectrum still have off-gage phenomenon on the high frequency .but when we identify frequency response function based on the EV model, the control spectrum can control reference spectrum which is in the $\pm 3 \mathrm{~dB}$ tolerance limit on the all frequency band, it is obvious that it can improve control precision control precision of random vibration experiment.

\section{Conclusion}

It puts forward a frequency response function estimation method based EV model, it improves the traditional random vibration control algorithm.EV model consider the noise of the systems' input terminal and output terminal at the same time, so the frequency response function is more accurate because of EV model. The experimental results of random vibration acceleration spectrum reappearance shows that within the same iteration number,,improved random vibration control algorithm can increase the control precision of the experiments.

\section{References}

[1] Zhang wei Chen, Hui jun YU .Vibration control status and progress[J] .Vibration and Shock .2009, 28(3): 73-77, 86, 200.

[2] Stroud R.C., Hamma G.A. and Underwood M. A et al. A Review of Multiax is/Multiexciter Vibration Technology[J]. Sound and Vibration. 1996, 30(4): $20 \sim 27$.

[3] Edwin A. Sloane. Vibration Control System [P]. United States Patent, 4989158. 1991-01-29.

[4] Underwood M. A and Keller T. Recent System Developments for Multi-actuator Vibration Control Systems [J]. Sound and Vibration. 2001, 35(10): 16 23

[5] UNDERWOOD M A. Adaptive control method for multiexciter sine tests [P]. United States, 5299459. 1994-04-05.

[6] Zhiqiang $\mathrm{Hu}$, Qingyan Fa, Baolin Hong.Random vibration test applied technology [M].Beijing China Metrology Publishing House.1996. 125-130.

[7] China Machinery Industry Federation. JB/T 8288-2001.People's Republic of China Machinery Industry Standard. Hydraulic vibration table.2001. 